\title{
Regimes of flux transport at microwave frequencies in nanostructured high- $T_{c}$ films
}

\author{
R. Wördenweber, ${ }^{*}$ E. Hollmann, J. Schubert, R. Kutzner, and G. Panaitov \\ Peter Grünberg Insitute (PGI) and JARA-Fundamentals of Future Information Technology, Forschungszentrum Jülich, \\ D-52425, Germany
}

(Received 14 November 2011; published 3 February 2012)

\begin{abstract}
We report on combined dc and microwave electronic measurements of magnetic flux transport in micron and submicron-patterned high- $T_{c}$ films. In a given temperature regime below the superconducting transition temperature $T_{c}$, the current-driven flux transport is restricted to flux motion guided by the submicron patterns. Via frequency-dependent measurements of the forward transmission coefficient $S_{21}$ it is demonstrated that the mechanism of the guided flux transport depends on the microwave frequency and the geometrical size of the superconducting structures. At low frequencies, flux is transported via Abrikosov vortices leading to additional microwave losses. Above a geometrically defined frequency, a different mechanism seems to be responsible for flux transport that does not contribute to the microwave losses and most likely represents a phase-slip type mechanism. The limiting vortex velocity obtained from the frequency dependence of the microwave properties agrees with the Larking-Ovchinnikov critical vortex velocity that is determined via dc pulse measurements. In spite of the change of mechanism, guidance of flux persists in these nanopatterns up to high frequencies of several GHz.
\end{abstract}

DOI: 10.1103/PhysRevB.85.064503

PACS number(s): 74.25.Uv, 74.72.-h, 78.70.Gq

\section{INTRODUCTION}

The understanding of flux transport in mesoscopic or even nanostructured superconducting systems that are exposed to microwave electric fields is not only of relevance for microwave fluxonic applications, it is also of interest for the general understanding of vortex matter and thus, high velocities. It has been demonstrated that vortex matter can be manipulated very efficiently via modification of the superconducting properties on a micrometer or nanometer scale. For instance, non-simply-connected micro- or nanostructures will cause long-range electronic or magnetic interactions between vortices and artificial structures. Various arrangements of possible micro- and nano-objects ranging from magnetic to nonmagnetic dots, ${ }^{1-4}$ completely etched holes (antidots), ${ }^{5-11}$ to partially etched holes (blind holes) ${ }^{12}$ are feasible and can lead to vortex pinning, trapping, annihilation, or guidance.

Especially the use of (arrays of) micron- or nanosize antidots has been proven to be a very effective way to manipulate flux in superconducting films and devices. In contrast to other pinning defects, which have to be of the size of the superconducting coherence length $\xi$, antidots with sizes much larger than $\xi$ will trap magnetic flux very effectively. ${ }^{9}$ The advances in lithography techniques and the possible use of antidots in applications (e.g., SQUIDs, ${ }^{13,14}$ vortex diodes, ${ }^{15-17}$ fluxonic microwave devices ${ }^{18}$ ) have led to a renewed interest in the research of superconducting films containing antidots or antidot lattices.

First indications for guidance of vortices by rows of antidots have been obtained from magneto-optic imaging of flux penetration in patterned, high- $T_{c}$ films. ${ }^{19}$ This was followed by a quantitative analysis of the guided motion measured via Hall-type experiments and explained by a vortex channel model. ${ }^{11}$ Recently it was demonstrated that magnetic flux can be guided by micrometer-size antidots structures in $\mathrm{YBa}_{2} \mathrm{Cu}_{3} \mathrm{O}_{7-\delta}$ (YBCO) even up to frequencies of at least $8 \mathrm{GHz} .{ }^{20}$ This demonstration is based on the vortex ratchet effect. Since this demonstration is important for the discussion below and represents a motivation for the research given in this paper, the main result and the schematic setup of the experiment are summarized in Fig. 1. In the temperature regime of high vortex mobility (reversible regime below $T_{c}$ ), vortex motion is stimulated by a microwave current and guided by rows of antidots. Due to the anisotropic (triangular) shape of the antidots the guided motion is rectified resulting in a dc voltage signal that is shown in the contour plot of Fig. 1. It was very surprising that in spite of the large distance $(4.3 \mu \mathrm{m})$ between adjacent antidots, rectified guidance of vortices persisted up to such high frequencies. It automatically led to the question of the mechanism of flux transport at high frequencies.

According to Larkin and Ovchinnikov the normal core of an Abrikosov vortex is expected to collapse at high vortex velocities. ${ }^{21}$ As a consequence microwave-driven Abrikosov vortex transport across a typical microwave-suitable structure (e.g., impedance matched stripline) with subcritical vortex velocity is expected to be limited to frequencies clearly below the $\mathrm{GHz}$ regime. Moreover, based on theoretical considerations (e.g., time-dependent Ginzburg-Landau theory) vortices moving in micron- or nanosize constrictions are expected to be modified. For instance, the vortex core of a vortex in the vicinity of the antidot will be deformed ${ }^{22,23}$ and, finally, a phase-slip type mechanism for flux transport is expected for flux transport in superconducting microbridges. ${ }^{24}$ Therefore these questions arise: (i) Which type of vortex is responsible for the guided flux transport at elevated frequencies $(\gg 1 \mathrm{GHz})$ and (ii) can we identify a transition from the classical flux transport via Abrikosov vortices at low frequencies to an alternative, perhaps novel type of flux transport at elevated frequencies?

In this paper, we experimentally demonstrate a change of the mechanism of flux transport at a geometrically defined frequency in the GHz frequency regime. The data are discussed in terms of theoretical predictions ${ }^{21}$ and compared with experimental data ${ }^{25,26}$ obtained for the critical vortex velocity. 


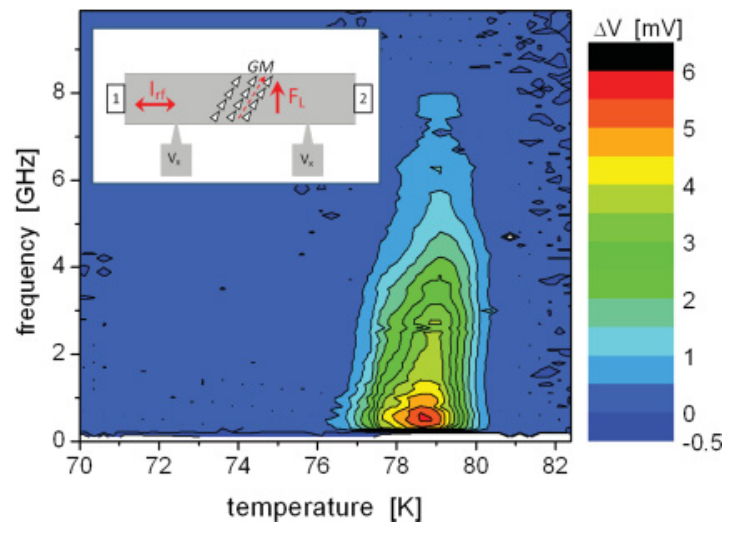

FIG. 1. (Color online) Demonstration of vortex manipulation up to $8 \mathrm{GHz}$ based on the ratchet effect in micropatterned high- $T_{c}$ microwave devices (Ref. 20). The inset shows a schematic drawing of a YBCO ratchet based on rows of triangular-shaped antidots. The microwave current is applied via ports 1 and 2, the vortex flow is rectified by the rows of triangular antidots, and the resulting dc voltage signal is recorded at the dc voltage contacts $V_{\mathrm{x}}$. The contour plot shows the dc voltage signal $\Delta V_{\mathrm{dc}}=V_{\mathrm{dc}}(-10 \mathrm{dBm})-V_{\mathrm{dc}}(-50$ $\mathrm{dBm})$ as function of temperature and applied microwave frequency. The arrows $I_{\mathrm{rf}}, F_{\mathrm{L}}$, and $\mathrm{GM}$ in the inset indicate the direction of microwave current, resulting Lorentz force acting on the vortices, and the rectified guided motion of vortices.

\section{SAMPLE PREPARATION AND EXPERIMENTAL SETUP}

$\mathrm{YBa}_{2} \mathrm{Cu}_{3} \mathrm{O}_{7-\delta}$ (YBCO) thin films (film thickness of $d \approx$ $50-120 \mathrm{~nm}$ ) are deposited via pulsed laser deposition or magnetron cathode sputtering on microwave-suitable substrates, i.e., $\mathrm{LaAlO}_{3}$ and $r$-cut sapphire with a 30-nm-thick $\mathrm{CeO}_{2}$ buffer. The films are patterned via $e$-beam lithography and ion-beam etching at $500 \mathrm{eV}$. Microwave striplines with a width of $400 \mu \mathrm{m}$ are patterned resulting in an microwave impedance of $50 \Omega$. Furthermore, an array consisting of rows of circular-shaped holes (antidots) is patterned into the stripline (see Fig. 2). In order to be able to distinguish between vortex motion along the Lorentz force and guided motion within the rows, the rows are tilted by $35^{\circ}$ with respect to the nominal direction of the Lorentz force. Furthermore, the experimental setup allows to simultaneously apply dc and microwave (0.4$6 \mathrm{GHz}$ ) currents.

In this paper, data of a reference sample (without antidots) and two samples (AA1 and AA2) with antidots are discussed. Sample AA1 is equipped with smaller antidots (nominal antidot radius $r_{a}=250 \mathrm{~nm}$ ) and antidot spacing (antidot separation within the rows $d_{\mathrm{aa}}=1 \mu \mathrm{m}$ ), whereas sample AA2 has larger antidots $\left(r_{a}=1 \mu \mathrm{m}\right)$ and antidot separation within the rows $\left(d_{\mathrm{aa}}=4 \mu \mathrm{m}\right)$. In both samples the distance between adjacent rows is $10 \mu \mathrm{m}$.

\section{EXPERIMENTAL RESULTS}

In general, microwave currents are relatively small. Therefore, the resulting Lorentz force is not capable of moving Abrikosov vortices over larger distances. In order to enable vortices to move over larger distance (e.g., throughout the sample or between antidots with larger antidot separation), a dc bias current is added to the microwave current. In our

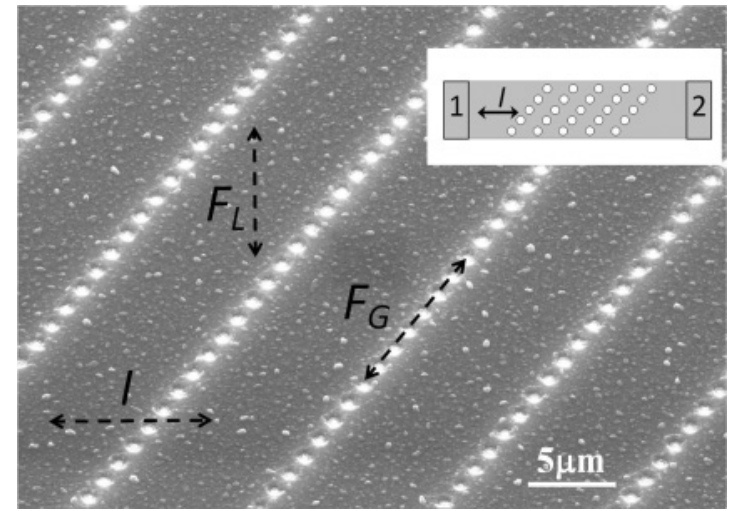

FIG. 2. Sketch of the YBCO stripline with antidots and current ports (inset) and microscopic image of sample AA1 $\left(r_{a}=250 \mathrm{~nm}\right.$, $d_{\mathrm{aa}}=1 \mu \mathrm{m}$ ). About 400 rows are positioned on a microwave stripline. The nominal directions of current ( $\mathrm{rf}$ and $\mathrm{dc}$ ), resulting Lorentz force, and guiding force are indicated by the arrows.

experiments the dc-bias current is typically five to ten times larger than the microwave current. We apply a microwave power of approximately $-10 \mathrm{dBm}$ at the sample resulting in a total microwave current of $\sim 1-2 \mathrm{~mA}$, whereas the $\mathrm{dc}$ bias is typically $5-10 \mathrm{~mA}$. Since the microwave current is mainly restricted to the edge of the stripline, ${ }^{27}$ it can locally approximate or even exceed the dc current density.

The aim of our experiments is to determine the microwave losses that are caused by vortex or flux motion in the superconducting regime, especially for the case of guided vortex or flux motion. This is achieved by comparing the microwave transmission at given frequency and microwave power $(-10 \mathrm{dBm})$ with and without additional dc bias current. The modification (namely the reduction) of the microwave forward transmission coefficient $S_{21}$ due to the dc bias provides a measure for the microwave loss due to current-driven vortex motion in the sample. $S_{21}=P_{2} / P_{1}$ describes the microwave response $P_{2}$ at the output (port 2) due to an input microwave signal $P_{1}$ at the input (port 1 ) at a given frequency (see, for instance, inset of Fig. 2). In the following, we mainly discuss the temperature and frequency dependence of the difference of microwave forward transmission coefficient $\Delta S_{21}=S_{21}\left(J_{\mathrm{dc}} \neq\right.$ $0)-S_{21}\left(J_{\mathrm{dc}}=0\right)$, where $J_{\mathrm{dc}}$ defines the dc bias current density. Different temperature regimes (R1-R4; see Figs. 3 and 4) can be identified for which different types of flux transport are present. Finally, we focus on the temperature regime R3 of guided flux motion. In this temperature regime, vortices or flux are moving from antidot to antidot within the rows, i.e., only in this temperature regime can vortices or flux motion in micron or submicron structures be studied.

Figure 3 shows the temperature dependence of $P_{2}$ (with and without dc current) and the resulting difference $\Delta S_{21}=S_{21}\left(J_{\mathrm{dc}} \neq 0\right)-S_{21}\left(J_{\mathrm{dc}}=0\right)$ for the reference sample. Neglecting the behavior in the normal state (here additional quasiparticle scattering leads to additional losses caused by the dc current), $S_{21}$ only differs in a small temperature regime (R1) just below the superconducting transition temperature $T_{c}=$ $90.1 \mathrm{~K}$. This is best visualized by considering the temperature dependence of $\Delta S_{21}$ [Fig. 3(b)] that displays a pronounced minimum in the temperature regime $\mathrm{R} 1$. We can exclude 

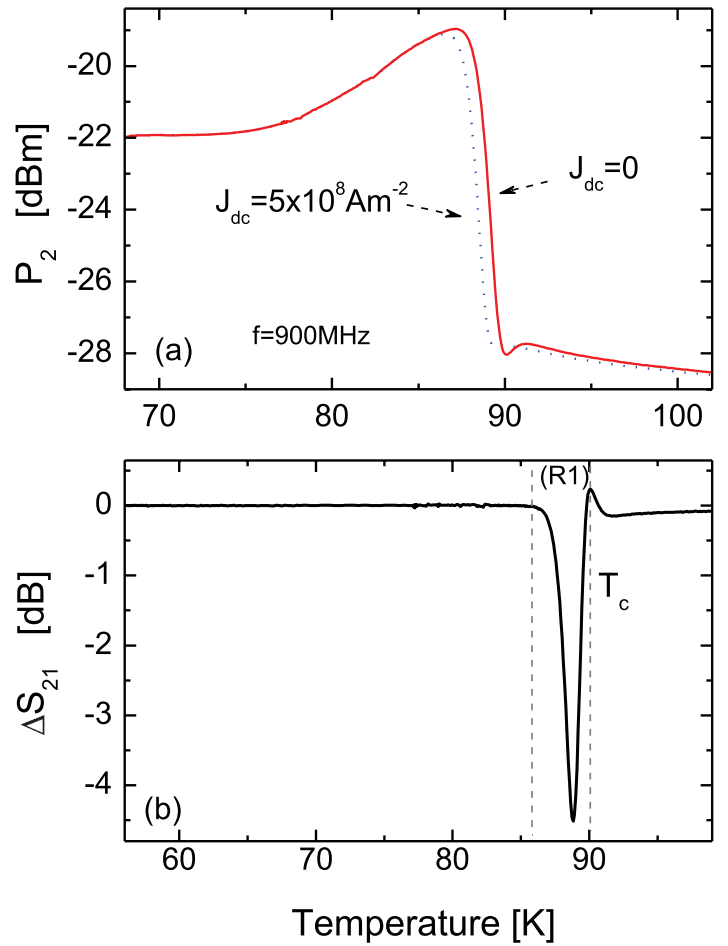

FIG. 3. (Color online) Temperature dependence of the output microwave power $P_{2}$ with and without dc bias (a) and the resulting $\Delta S_{21}=S_{21}\left(J_{\mathrm{dc}} \neq 0\right)-S_{21}\left(J_{\mathrm{dc}}=0\right)$ of the reference sample without antidots. The film thickness is $120 \mathrm{~nm}$, the microwave power at the sample is $-10 \mathrm{dBm}$, and a magnetic field of $1 \mathrm{mT}$ is applied normal to the film's surface. The transition temperature $\left(T_{c}=90.1 \mathrm{~K}\right)$, dc bias $\left(0\right.$ and $\left.5 \times 10^{8} \mathrm{Am}^{-2}\right)$, and the frequency $(900 \mathrm{MHz})$ are given in the figure. Furthermore, a temperature regime (R1) is marked that will be discussed in the context of the regimes R1-R4 given in Fig. 4.

experimental artifacts (e.g., self-heating induced by the dc current close to $T_{c}$ ) to cause the minimum. Furthermore, from microwave transmission experiments on YBCO coplanar resonators in magnetic fields ${ }^{28}$ we conclude that the additional microwave loss is mainly caused by flux motion. Close to $T_{c}$, flux pinning is negligible (reversible regime) and flux lines can be moved throughout the stripline. Due to the additional dc current additional loss occurs since vortices are moved into the regime of large microwave current (edge of the stripline) where they are exposed to large microwave driving forces. ${ }^{18}$ With reducing temperature flux pinning increases, finally vortices will be pinned and only oscillate within their potential well. Consequently, $\Delta S_{21}$ shows only a pronounced minimum below $T_{c}$ and is zero for all temperatures below regime R1.

The temperature dependence of $\Delta S_{21}$ obtained for striplines with antidots is more complex. We will discuss the behavior in terms of the different temperature regimes $\mathrm{R} 1-\mathrm{R} 4$ given in Fig. 3:

Regime R1. For both samples a pronounced minimum is observed for the temperature regime R1 just below $T_{c}$. Due to the sub- $\mu \mathrm{m}$ patterning this regime is more extended and the minimum is less pronounced for the smaller antidot array [sample AA1, Fig. 4(a)]. In the case of the larger array [sample AA2, Fig. 4(b)] the width and depth of this minimum are similar to that of the reference sample [Fig. 3(a)]. Since the

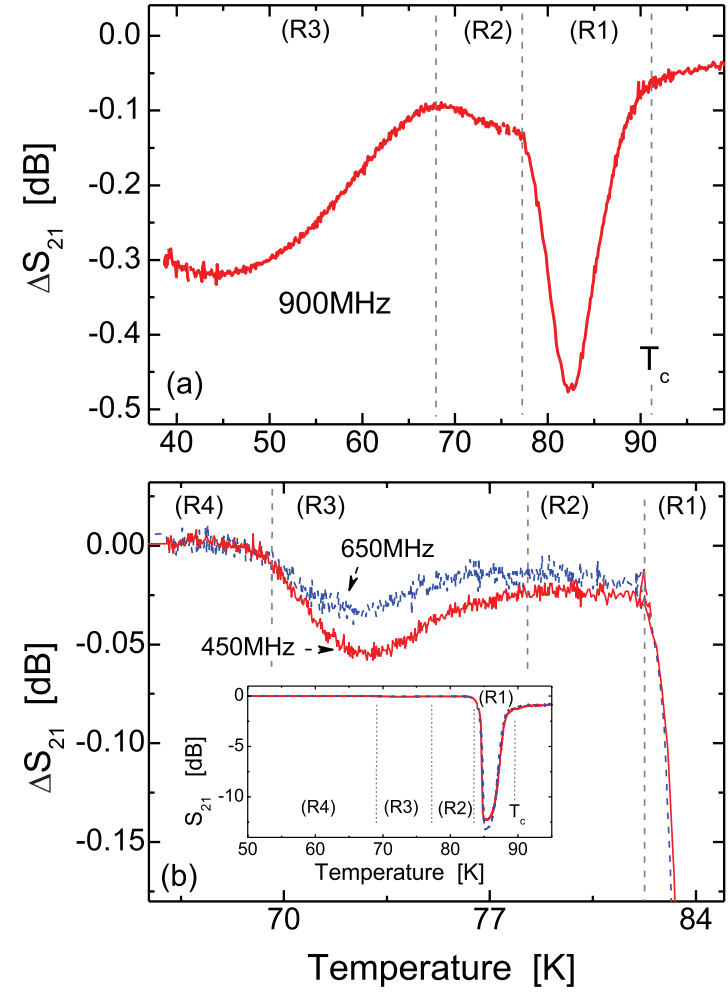

FIG. 4. (Color online) Temperature dependence of the difference of the forward transmission coefficient $\Delta S_{21}=S_{21}\left(J_{\mathrm{dc}} \neq 0\right)-S_{21}\left(J_{\mathrm{dc}}\right.$ $=0)$ for two striplines with rows of antidots: (a) sample $\operatorname{AA} 1\left(r_{a}=\right.$ $\left.250 \mathrm{~nm}, d_{\mathrm{aa}}=1 \mu \mathrm{m}\right)$ and (b) sample AA2 $\left(r_{a}=1 \mu \mathrm{m}, d_{\mathrm{aa}}=4 \mu \mathrm{m}\right)$. The film thicknesses are $50 \mathrm{~nm}$ (AA1) and $120 \mathrm{~nm}$ (AA2), the $T_{c}$ values are indicated (right limit of regime R1), the microwave power at the sample is $-10 \mathrm{dBm}$, a magnetic field of $1 \mathrm{mT}$ is applied normal to the film's surface, and the dc bias is zero, $5 \times 10^{8} \mathrm{Am}^{-2}$ (AA1), and $8 \times 10^{8} \mathrm{Am}^{-2}$ (AA2), respectively. Different temperature regimes $\mathrm{R} 1-\mathrm{R} 4$ characterizing different types of vortex motion are indicated in the figures. The inset in (b) shows the original transmission coefficient $S_{21}$ (with and without dc bias) as function of temperature for sample AA2.

vortex-defect interaction (including vortex-antidot interaction) is small close to $T_{c}$ vortices move freely in the stripline. Moreover, since the rows of antidots are tilted, the vortices are not guided by the antidots. Both effects have, for instance, been demonstrated by Hall-type measurements. ${ }^{11}$ Therefore, in this temperature regime the origin of the additional microwave loss is identical to that observed for the reference sample, i.e., microwave-generated and dc-current-assisted vortex motion in the superconducting stripline (not guided by antidots) leads to the additional loss that is visible in $\Delta S_{21}$ below $T_{c}$.

Regime $R 2$. In this regime flux pinning sets in. With decreasing temperature it increases and $\Delta S_{21}$ tends to zero. This again is identical to the behavior observed for the reference sample at temperatures just below R1.

Regime R3. In this regime a behavior different to that of the reference sample is observed. A second minimum in $\Delta S_{21}$ is present for our samples with rows of antidots. The width and depth of the second minimum strongly depend on the separation of the antidots. The minimum is more pronounced for the sample AA1 [antidot array with smaller periodicity, 
Fig. 4(a)]. It is barely visible for sample AA2 [array with larger periodicity, Fig. 4(b)]. From Hall measurements we know that this second minimum is caused by guided motion of flux, i.e., vortex or flux motion within the rows of antidots. ${ }^{11,29}$ At lower temperatures the vortex-antidot interaction becomes larger. If the spacing between adjacent antidots is small enough, vortices will move from antidot to antidot even if the directing of motion is not identical to the direction of the driving Lorentz force.

Regime R4. Finally, at very low temperatures the vortexantidot interaction is too large and flux is trapped in the antidots. As a result $\Delta S_{21}$ is zero. Additional measurements for lower temperatures that are not shown in Fig. 4 demonstrate that $\Delta S_{21} \approx 0$ for sample AA1 at 4.2 and $10 \mathrm{~K}$.

Obviously it is most interesting to analyze the guided vortex or flux motion of regime R3 in more detail since it represents the regime of vortex or flux transport in micron or even submicron superconducting structures. For this we recorded the frequency dependence of $\Delta S_{21}$ for the different samples in the frequency regime $0.4-6 \mathrm{GHz}$.

Excluding the transition regime $\mathrm{R} 1, \Delta S_{21} \approx 0$ for the all frequencies for the reference sample. The samples with antidots (AA1 and AA2) show no or very little frequencydependent additional losses in the temperature regimes $\mathrm{R} 2$ and R4, respectively [see Fig. 4(b)]. However, a significant frequency dependence of $\Delta S_{21}$ is observed for the regime R3 of guided flux motion [Fig. 4(b)]. Figure 5 shows the frequency dependence of $\Delta S_{21}$ for the two different samples with antidots in the regime of guided flux motion (R3) in more detail. At low frequencies $\Delta S_{21}$ is clearly nonzero and negative, i.e., additional microwave losses due to vortex motion are present. These losses decrease with increasing frequency and finally vanish at high frequencies. Neglecting resonance effects in the experimental setup, the maximum frequency up to which losses are observed are $f_{\max } \sim 2.9 \mathrm{GHz}$ and $\sim 1.0 \mathrm{GHz}$ for sample AA1 and AA2, respectively.

\section{DISCUSSION}

In general, the interpretation of microwave losses due to vortex motion has proven to be quite complex. It has been demonstrated that vortex-generated microwave losses occur only when vortices are present in areas of the sample that carry large microwave current densities. ${ }^{18}$ Obviously the interpretation becomes even more complicated in the case of vortex motion in patterned superconducting systems, e.g., superconducting films with an antidot lattice.

On the one hand, modifications of the vortex properties due to their vicinity to antidots have to be considered. For larger antidot distances this might lead to a deformation of the vortex core of an Abrikosov vortex..$^{22,23}$ However, for smaller antidot distances the superconductor between two adjacent antidots can be considered to act like a microbridge ${ }^{11}$ and a phase-slip type of flux transport is expected. ${ }^{24,30}$ Since a phase slip would exclude the generation of a normal core (i.e., no Abrikosov vortex) flux pinning would be absent for the flux transport across the microbridge and, moreover, it is expected to lead to a flux transport that does not generate additional microwave loss. ${ }^{18}$ The latter is what we observe in our experiment at high frequencies.
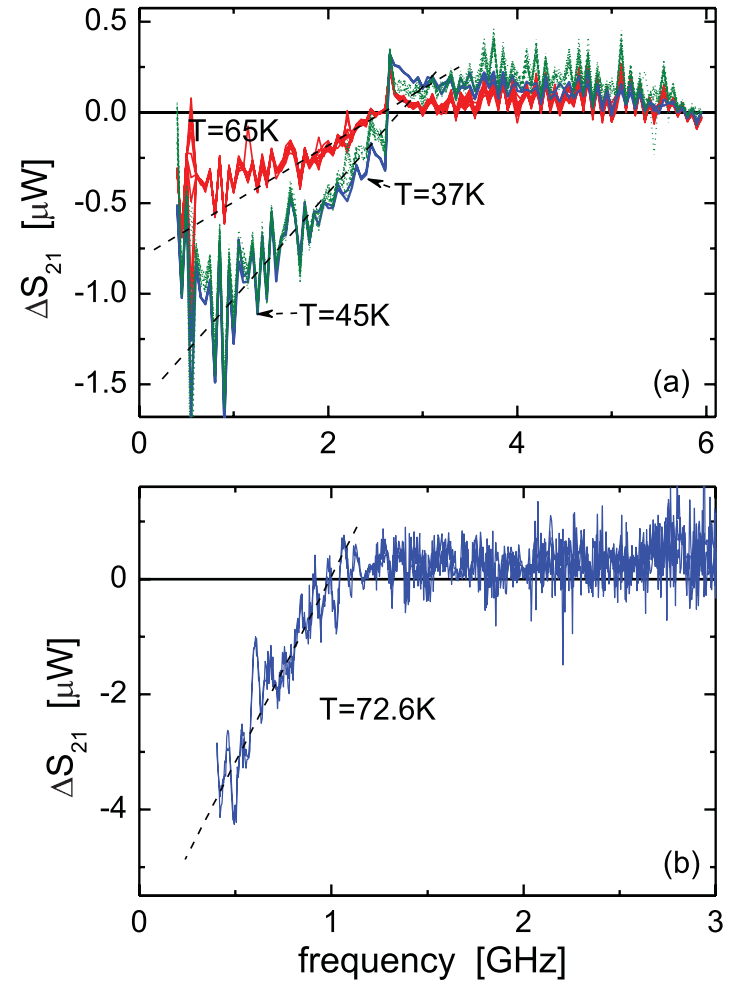

FIG. 5. (Color online) Frequency dependence of $\Delta S_{21}$ for the sample AA1 (a) and AA2 (b). The microwave power at the sample is $-10 \mathrm{dBm}$, dc bias is $5 \times 10^{8} \mathrm{Am}^{-2}$ (AA1) and $8 \times 10^{8} \mathrm{Am}^{-2}$ (AA2), a magnetic field of $1 \mathrm{mT}$ is applied normal to the films surface, and the temperatures are given in the figures.

On the other hand, flux transport between antidots and at microwave frequencies implies large vortex velocities. One should note that flux motion guided by an antidot lattice automatically implies a motion of flux quanta between adjacent antidots. The impact of the velocity on the Abrikosov vortex is described by Larkin and Ovchinnikov. ${ }^{21}$ Since we have to assume that at low frequencies the flux transport takes place in form of the motion of Abrikosov vortices (which leads to additional microwave loss), it is feasible that at the maximum frequency $f_{\max }$ the critical velocity is reached (at which the normal core of the vortex collapses) and a transition to phase-slip-type flux transport takes place.

Combining these different aspects of vortex matter and our experimental results, we can devise the following model that describes the guided flux transport in our micronand submicron-patterned superconductor at microwave frequencies:

(i) Guided vortex or flux transport (regime R3) implies motion of flux quanta between adjacent antidots. The flux transport does not necessarily have to take place in the form of Abrikosov vortices. Guided flux motion is demonstrated for frequencies up to $8 \mathrm{GHz}$ (see Fig. 1). However, this limitation might be caused by the experimental setup that was designed for measurements up to a maximum frequency of $10 \mathrm{GHz}$.

(ii) The driving force for the guided flux transport is provided by the Lorentz force that is caused by the combined action of the dc and microwave currents. Although the current oscillates between the minimum current 
$I_{\min }=I_{\mathrm{dc}, \max }-I_{\mathrm{ac}, \max }$ and maximum current $I_{\max }=$ $I_{\mathrm{dc}, \max }+I_{\mathrm{ac}, \max }$, the time-averaged Lorentz force is independent of the frequency for given dc-bias and microwave currents (small variations due to resonances in the measurement circuit can be neglected).

(iii) At low frequencies the transport takes place in the form of Abrikosov vortices. The current-time profile resembles a current ramp from $I_{\min }$ to $I_{\max }$ followed by a drop to the minimum current. As a consequence the Lorentz force is slowly ramped up, and magnetic flux is pushed out of the antidots and Abrikosov vortices with a normal core nucleate. The dc-bias-assisted vortex transport leads to the accelerated vortex transport (current ramp) between adjacent antidots and is afflicted with an additional microwave loss. ${ }^{18}$

(iv) With increasing frequency the angle of the current ramp becomes steeper which leads to an increased acceleration of the nucleated Abrikosov vortices. As a consequence and according to the Larkin-Ovchinnikov theory, ${ }^{21}$ the shrinkage of the normal core increases with increasing frequency. This automatically implies a reduction of the viscous damping coefficient for vortex motion and an increase of the vortex velocity. ${ }^{21}$ It is most likely that the additional microwave losses are also decreasing with decreasing normal core, ${ }^{18}$ i.e., with increasing frequency (see also Fig. 5).

(v) Finally, at a high enough frequency the critical velocity $v^{*}$ might be reached. ${ }^{21}$ The normal core of the vortex would collapse and the flux would be transferred in a phase-slip-type mechanism without causing any additional microwave loss (see Fig. 5). This would have a number of consequences; for instance, flux pinning would not be applicable anymore and flux transfer at velocities exceeding the critical velocity would be possible. As a consequence, flux transfer between adjacent antidots would be feasible even at very high frequencies as demonstrated in the experiment (see Fig. 1).

In order to test this model and especially assumption (v), we analyze the transition from the lossy mechanism (Abrikosov vortices) to a loss-free mechanism (phase-slip-type mechanism) in terms of the Larkin-Ovchinnikov theory. If we assume that the limiting frequency $f_{\max }$ defines a limit of the mobility of Abrikosov vortices in these nanostructured systems, we can estimate a maximum velocity $v_{\max }$ for Abrikosov vortices to be on the order of

$$
v_{\max } \cong 2 f_{\max } \cdot d_{\mathrm{aa}},
$$

where $d_{\mathrm{aa}}$ (distance between adjacent antidots) defines the distance the vortex travels in case of guided vortex motion. Inserting the values for $f_{\max }$ and $d_{\mathrm{aa}}$ for samples AA 1 and AA2, a maximum velocity of $v_{\max } \approx 5.8-8 \mathrm{~km} / \mathrm{s}$ is obtained for Abricosov vortices motion in our nanostructured samples. This value is on the order of experimental data obtained for the critical velocity $v^{*}$ that is determined via dc pulse measurements on YBCO films. ${ }^{25,26}$ Thus, our values for the maximum velocity $v_{\max }$ seem to agree with the vortex critical velocity $v^{*}$ obtained by other experimental techniques. Moreover, based on Eliashberg's ideas on nonequilibrium effects in superconductors, Larkin and Ovchinnikov predicted that a nonequilibrium distribution and relaxation rate of the normal charge carriers (quasiparticles) develops during the motion of vortices at high velocities. According to the theory,

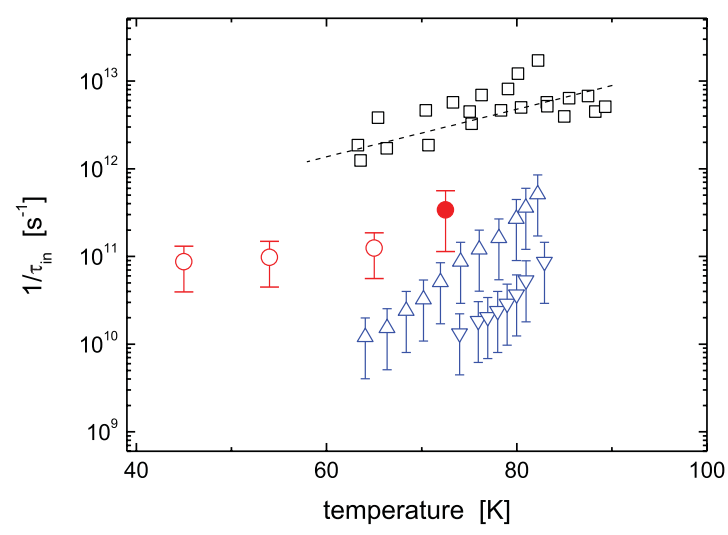

FIG. 6. (Color online) Temperature dependence of the inelastic quasiparticle scattering time $\tau_{\text {in }}$ obtained for guided vortex motion in nanostructured YBCO striplines AA1 (open circles) and AA2 (solid circle). For comparison zero-field microwave experiments (squares) (Refs. 30 and 31) and dc pulse measurements (triangles) (Refs. 25 and 26) on YBCO thin films are included.

the viscous damping coefficient at a vortex velocity $v$ is given by $^{21}$

$$
\eta(v)=\eta(0)\left[1+\left(\frac{v}{v^{*}}\right)^{2}\right]^{-1},
$$

resulting in an inelastic quasiparticle scattering time:

$$
\tau_{\text {in }}=\frac{D \cdot \sqrt{14 \varsigma(3)\left(1-T / T_{c}\right)}}{\pi}\left(\frac{1}{v^{*}}\right)^{2},
$$

with $\zeta(x)$ denoting the Riemann-zeta function, $D=v_{F} 1_{o} / 3$ the quasiparticle diffusion coefficient, $v_{F}$ the Fermi velocity, and $l_{o}$ the electron mean free path. Inserting reasonable values for $v_{F}(=200-500 \mathrm{~km} / \mathrm{s})$ and $l_{o}(=4-8 \mathrm{~nm})$ (Ref. 31), the inelastic quasiparticle scattering time can be estimated for our experiment by inserting $v_{\max }$ into Eq. (3). Figure 6 represents the resulting inelastic quasiparticle scattering time obtained for our samples. Please note that we can only determine data for the temperature regime of guided flux motion. For comparison, experimental data obtained via pulse measurements ${ }^{25,26}$ and microwave experiments ${ }^{32,33}$ are added. At temperatures between 40 and $70 \mathrm{~K}$, inelastic quasiparticle scattering times of $\tau_{\text {in }} \approx 10^{-11} \mathrm{~s}$ are obtained; $\tau_{\text {in }}$ decreases slightly with temperature. These values range between those obtained from dc pulse measurements and zero-field microwave experiments.

\section{SUMMARY}

It has been demonstrated that guided flux motion in high- $T_{c}$ films with rows of antidots persists up to frequencies of several $\mathrm{GHz}$ (here $8 \mathrm{GHz}$ ). However, considering the antidot-antidot distance a flux transport via Abrikosov vortices is very unlikely for these high frequencies. With the analysis of the frequency dependence of the guided vortex motion we could demonstrate that there exist two different regimes of flux transport in microand submicron-structured superconductors. Flux transport via Abrikosov vortices is only present up to a geometrically defined maximum frequency $f_{\max }$ that most likely represents the critical velocity predicted by Larkin and Ovchinnikov. ${ }^{21}$ 
This type of flux transport leads to additional microwave losses. At the characteristic frequency $f_{\max }$ the additional microwave losses vanish and the mechanism of flux transport cannot be described by the classical transport of Abrikosov vortices any more. Most likely a phase-slip type of flux transport that is known to take place in microbridges and that does not cause additional microwave losses takes over. Nevertheless, flux manipulation in nanostructured high- $T_{c}$ films persists to high frequencies at which the vortex velocity clearly exceeds the critical velocity. Thus, in spite of the change of the mechanism of flux transport, nanostructured high- $T_{c}$ films might have the potential to be excellent candidates for fluxonic devices working in the $\mathrm{GHz}$ regime.

\section{ACKNOWLEDGMENTS}

The authors would like to thank A. Offenhäuser, V. R. Misko, H. P. Bochem, M. Nonn, and B. T. Chung for their valuable support. This work was supported by the ESF program Nanoscience and Engineering in Superconductivity-NES. *r.woerdenweber@fz-juelich.de

${ }^{1}$ J. I. Martín, M. Vélez, J. Nogués, and I. K. Schuller, Phys. Rev. Lett. 79, 1929 (1997).

${ }^{2}$ M. Andersson, J. C. Cuevas, and M. Fogelström, Physica C 367, 117 (2002).

${ }^{3}$ M. Lange, M. J. Van Bael, Y. Bruynseraede, and V. V. Moshchalkov, Phys. Rev. Lett. 90, 197006 (2003).

${ }^{4}$ M. P. González, E. Hollmann, and R. Wördenweber, J. Appl. Phys. 102, 063904 (2007).

${ }^{5}$ M. Baert, V. V. Metlusko, R. Jonckheere, V. V. Moshchalkov, and Y. Bruynseraede, Phys. Rev. Lett. 74, 3269 (1995).

${ }^{6}$ V. V. Metlushko, M. Baert, R. Jonckheere, V. V. Moshchalkov, and Y. Bruynserade, Solid State Commun. 91, 331 (1994).

${ }^{7}$ E. Rosseel, M. van Bael, M. Baert, R. Jonckheere, V. V. Moshchalkov, and Y. Bruynserade, Phys. Rev. B 53, R2983 (1996).

${ }^{8}$ V. V. Moshchalkov, M. Baert, V. V. Metlushko, E. Rosseel, M. J. van Bael, K. Temst, R. Jonckheere, and Y. Bruynserade, Phys. Rev. B 54, 7385 (1996).

${ }^{9}$ A. M. Castellanos, R. Wördenweber, G. Ockenfuss, A. v.d. Hart, and K. Keck, Appl. Phys. Lett. 71, 962 (1997).

${ }^{10}$ L. van Look, E. Rosseel, M. J. van Bael, K. Temst, V. V. Moshchalkov, and Y. Bruynseraede, Phys. Rev. B 60, R6998 (1999).

${ }^{11}$ R. Wördenweber, P. Dymashevski, and V. R. Misko, Phys. Rev. B 69, 184504 (2004).

${ }^{12}$ A. Bezryadin and B. Pannetier, J. Low Temp. Phys. 98, 251 (1995).

${ }^{13}$ P. Selders and R. Wördenweber, Appl. Phys. Lett. 76, 3277 (2000).

${ }^{14}$ R. Wördenweber and P. Selders, Physica C 366, 135 (2002).

${ }^{15}$ J. E. Villegas, S. Savel'ev, F. Nori, E. M. Gonzalez, J. V. Anguita, R. García, and J. L. Vicent, Science 302, 1188 (2003).

${ }^{16}$ B. Y. Zhu, F. Marchesoni, V. V. Moshchalkov, and F. Nori, Phys. Rev. B 68, 014514 (2003).

${ }^{17}$ J. Van de Vondel, C. C. de Souza Silva, B. Y. Zhu, M. Morelle, and V. V. Moshchalkov, Phys. Rev. Lett. 94, 057003 (2005); C. C. de Souza Silva, J. Van de Vondel, B. Y. Zhu, M. Morelle, and V. V. Moshchalkov, Phys. Rev. B 73, 014507 (2006).

${ }^{18}$ P. Lahl and R. Wördenweber, Appl. Phys. Lett. 81, 505 (2002).

${ }^{19}$ M. Pannetier, R. J. Wijngaarden, I. Fløan, J. Rector, R. Griessen, P. Lahl, and R. Wördenweber, Phys. Rev. B 67, 212501 (2003).
${ }^{20} \mathrm{R}$. Wördenweber, in Nanoscience and Engineering in Superconductivity, edited by V. V. Moshchalkov, R. Wördenweber, and W. Lang (Springer Verlag, Heidelberg,2010), p. 25.

${ }^{21}$ A. I. Larkin and Yu. N. Ovchinnikov, Zh. Eksp. Teor. Fiz. 68, 1915 (1975) [Sov. Phys. JETP 41, 960 (1976)].

${ }^{22}$ D. J. Priour Jr. and H. A. Fertig, Phys. Rev. B 67, 054504 (2003).

${ }^{23}$ V. M. Fomin and R. Wördenweber, Proc. SPIE 7364, 73640 (2009).

${ }^{24}$ K. K. Likharev and L. A. Yakobson, Sov. Phys. Tech. Phys. 20, 950 (1976).

${ }^{25}$ S. G. Doettinger, R. P. Huebener, R. Gerdemann, A. Kühle, S. Anders, T. G. Träuble, and J. C. Villégier, Phys. Rev. Lett. 73, 1691 (1994); Physica C 235-240, 3179 (1994).

${ }^{26}$ P. Lahl and R. Wördenweber, Appl. Supercond. 1997, 77 (1997).

${ }^{27}$ I. B. Vendik, O. G. Vendik, and D. I. Kaparkov, in Superconductor Microwave Technology, Part II: Superconducting Microwave Circuits, edited by E. Kollberg (Chalmers University of Technology, Göteborg, Sweden, 1996).

${ }^{28} \mathrm{R}$. Wördenweber, in Vortex Matter and Superconducting Electronic Devices, edited by A. V. Narlikar, High Temperature Superconductivity, Vol. 2 (Springer Verlag, Berlin-Heidelberg, 2004), p. 363.

${ }^{29}$ R. Wördenweber, E. Hollmann, J. Schubert, R. Kutzner, and Ajay Kumar Ghosh, Appl. Phys. Lett. 94, 202501 (2009).

${ }^{30}$ J. Schneider, H. Kohlstedt, and R. Wördenweber, Appl. Phys. Lett. 63, 2426 (1993); J. Schneider, M. Mück, and R. Wördenweber, ibid. 65, 2475 (1994).

${ }^{31}$ M. De La Pierre, S. Cagliero, A. Agostino, G. C. Gazzadi, and M. Truccato, Supercond. Sci. Technol. 22, 045011 (2009); A. P. Chernyaev, M. Chiba, A. L. Karuzskii, A. N. Lykov, V. N. Murzin, A. V. Perestoronin, and Yu.V. Vishnyakov, J. Phys. Chem. Solids 69, 3313 (2008); P. Seidel, M. Grajcar, A. Plecenik, and R. Hlubina, Physica B 218, 224 (1996); T. Oguchi, Phys. Rev. B 51, 1385 (1995); A. Hadni, X. Gerbaux, and J. C. Villegier, Phys. Status Solidi B 175, 409 (1993).

${ }^{32}$ F. Gao, J. W. Kruse, C. E. Platt, M. Feng, and M. V. Klein, Appl. Phys. Lett. 63, 2274 (1993).

${ }^{33}$ D. A. Bonn, Ruixing Liang, T. M. Riseman, D. J. Baar, D. C. Morgan, Kuan Zhang, P. Dosanjh, T. L. Duty, A. MacFarlane, G. D. Morris, J. H. Brewer, W. N. Hardy, C. Kallin, and A. J. Berlinsky, Phys. Rev. B 47, 11314 (1993). 